\title{
Subplate Neurons Promote Spindle Bursts and Thalamocortical Patterning in the Neonatal Rat Somatosensory Cortex
}

\author{
Else A. Tolner, ${ }^{1,2 \star}$ Aminah Sheikh, ${ }^{3 *}$ Alexey Y. Yukin, ${ }^{1}$ Kai Kaila, ${ }^{1,2}$ and Patrick 0. Kanold ${ }^{3}$ \\ ${ }^{1}$ Department of Biological and Environmental Sciences and ${ }^{2}$ Neuroscience Center, University of Helsinki, 00014 Helsinki, Finland, and ${ }^{3}$ Department of \\ Biology, University of Maryland, College Park, Maryland 20742
}

Patterned neuronal activity such as spindle bursts in the neonatal cortex is likely to promote the maturation of cortical synapses and neuronal circuits. Previous work on cats has shown that removal of subplate neurons, a transient neuronal population in the immature cortex, prevents the functional maturation of thalamocortical and intracortical connectivity. Here we studied the effect of subplate removal in the neonatal rat primary somatosensory cortex (S1). Using intracortical EEG we show that after selective removal of subplate neurons in the limb region of S1, endogenous and sensory evoked spindle burst activity is largely abolished. Consistent with the reduced in vivo activity in the $S 1$ limb region, we find by in vitro recordings that thalamocortical inputs to layer 4 neurons are weak. In addition, we find that removal of subplate neurons in the $S 1$ barrel region prevents the development of the characteristic histological barrel-like appearance. Thus, subplate neurons are crucially involved in the generation of particular types of early network activity in the neonatal cortex, which are an important feature of cortical development. The altered EEG pattern following subplate damage could be applicable in the neurological assessment of human neonates.

\section{Introduction}

Neuronal activity influences circuit development (Katz and Shatz, 1996; Erzurumlu and Kind, 2001; Zhang and Poo, 2001; Feldman and Brecht, 2005; Gonzalez-Islas and Wenner, 2006; Inan and Crair, 2007; Xu et al., 2011). A characteristic feature of neuronal activity in the immature mammalian nervous system are spontaneous, recurring network events that are present before the development of sensory driven activity (Yuste and Denk, 1995; Garaschuk et al., 2000; Ben-Ari, 2001; Adelsberger et al., 2005; Calderon et al., 2005; Vanhatalo et al., 2005). In preterm babies, the most prominent cortical EEG features are spontaneous activity transients characterized by slow waves nesting higher frequency oscillations (Vanhatalo et al., 2002). In the immature rodent somatosensory and visual cortex, around the end of the

Received March 25, 2011; revised 0ct. 31, 2011; accepted Nov. 18, 2011.

Author contributions: E.A.T., A.S., K.K., and P.O.K. designed research; E.A.T., A.S., A.Y.Y., K.K., and P.O.K. performed research; E.A.T., K.K., and P.O.K. contributed unpublished reagents/analytic tools; E.A.T., A.S., A.Y.Y., K.K., and P.O.K. analyzed data; E.A.T., A.S., K.K., and P.O.K. wrote the paper.

This work was supported by the Academy of Finland (E.A.T., K.K.), the Jane and Erkko Foundation, the Sigrid Jusélius Foundation (K.K.), the Finnish Cultural Foundation (A.Y.Y.), NIH Grant R01DC009607 (P.O.K.), and the International Cerebral Palsy Research Foundation (P.O.K.). K.K. is a member of the Finnish Center of Excellence in Molecular and Integrative Neuroscience Research. We thank Matias Palva and Anton Tokariev for scripts for data analysis; Kailin Hsu, Sreya Sinha, and Justin Penzenstadler for histological assistance; Akash Datwani and Carla Shatz for help with pilot experiments; Juha Voipio, Sampsa Vanhatalo, and Sebastian Schuchmann for helpful discussions; and Paul Watkins, Kevin Donaldson, and Sreya Sinha for help with analysis.

*E.A.T. and A.S. contributed equally to this work.

Correspondence should be addressed to Patrick 0. Kanold, Department of Biology, 1116 Biosciences Research Building, University of Maryland, College Park, MD 20742. E-mail: pkanold@umd.edu.

E. A. Tolner's present address: Department of Neurology and Department of Human Genetics, Leiden University Medical Centre, 2300RC Leiden, the Netherlands.

DOI:10.1523/JNEUROSCI.1538-11.2012

Copyright $\odot 2012$ the authors $\quad 0270-6474 / 12 / 310692-11 \$ 15.00 / 0$ first postnatal week, endogenous patterned activity is characterized mainly as recurrent spindle bursts (Khazipov et al., 2004; Bernard et al., 2005; Hanganu et al., 2006; Minlebaev et al., 2009; Yang et al., 2009; Colonnese and Khazipov, 2010; Mohns and Blumberg, 2010; Seelke and Blumberg, 2010). The shared characteristics of human preterm activity transients and rodent spindle bursts suggest that they are homologous functional features of the immature cortex (Milh et al., 2007; Minlebaev et al., 2009; Vanhatalo and Kaila, 2010). GABAergic and glutamatergic signaling underlies cortical spindle bursts (Khazipov et al., 2004; Minlebaev et al., 2007; Yang et al., 2009), but the circuitry that drives and controls them is unknown.

Subplate neurons (SPNs) are a transient population of neurons of the immature neocortex, located in the developing white matter (Kostovic and Rakic, 1980; Kanold and Luhmann, 2010). SPNs are innervated by thalamic axons before these grow into cortical layer 4, while SPNs provide excitatory input to layer 4 (Piñon et al., 2009; Zhao et al., 2009; Kanold and Luhmann, 2010). Thus SPNs are a transient relay and processing station for thalamic input and might be involved in the generation of cortical spindle bursts. First, SPNs have intrinsic oscillatory properties (Hirsch and Luhmann, 2008), are thought to support the propagation of pharmacologically evoked intracortical activity waves (Dupont et al., 2006), and might be active during spindle bursts (Yang et al., 2009). Second, SPNs are intimately associated with thalamocortical circuits, which might be involved in the generation of spindle bursts. Because spontaneous activity in the primary somatosensory cortex (S1) limb region is characterized mainly by spindle bursts (Khazipov et al., 2004; Yang et al., 2009) we first investigated the effect of SPN removal in the limb region. 

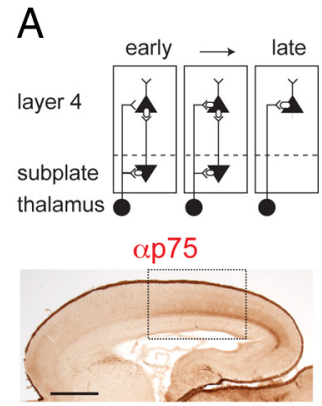

C
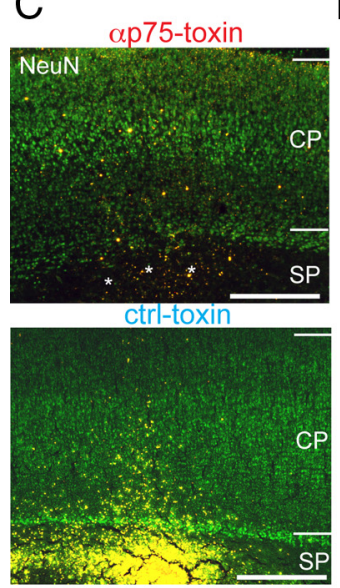

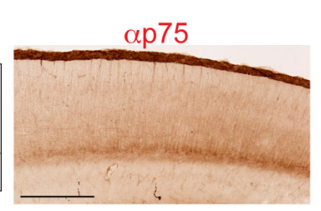

NeuN

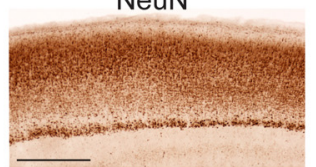

$\mathrm{D}$

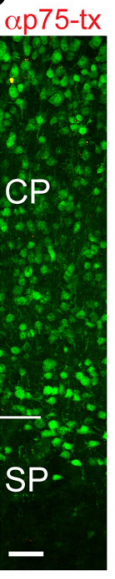

B

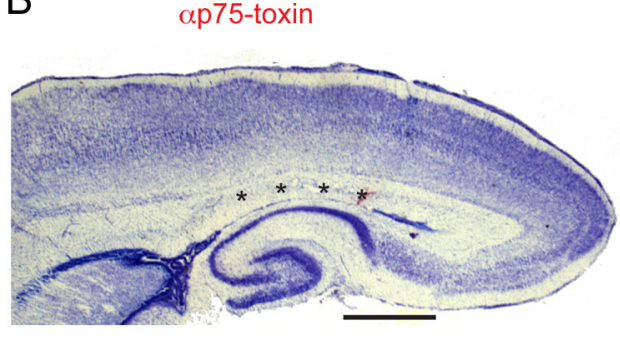

E

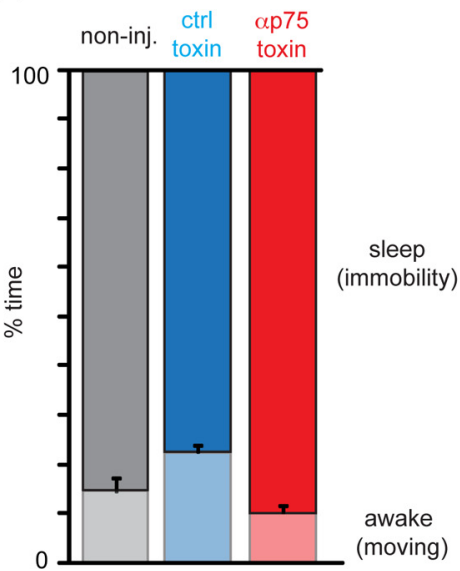

Figure 1. Selective removal of SPNs in rat S1.A, Schematic of subplate circuits (top left) and location of the subplate in rat. Distribution of $p 75$ in P0 rat brain as shown by immunolabeling using the antibody 192-lgG ( $\alpha$-p75) that is part of the immunotoxin used for SPN targeting. Bottom left, Low-power image. S1 marked by rectangle. Scale bar, $1 \mathrm{~mm}$. Top right, Detail of the S1/parietal cortex region depicted by the rectangle in low-power image. Scale bar, $500 \mu \mathrm{m}$. Bottom right, NeuN-stained adjacent brain section showing the subplate zone below the cortical plate. Deep white matter SPNs are poorly labeled by NeuN. Scale bar, $500 \mu \mathrm{m}$. B-D, Focal injections of anti-rat p75-immunotoxin at the level of SPN in P0 -P1 rats cause a highly selective ablation, leaving neurons in the overlying cortical plate intact. Niss ( $\boldsymbol{B}$ )- and $\operatorname{NeuN}(\boldsymbol{C}, \boldsymbol{D}$, labeled in green)-stained sections from a rat that received focal injections of anti-rat $\mathrm{p} 75$-immunotoxin $(\boldsymbol{B}$, and top in $\boldsymbol{C}$ ) in the $S 1$ cortex limb region at P1. The area of SPN ablation, marked by ***, covers an area of $\sim 1-1.5 \mathrm{~mm}$ in the mediolateral and anteroposterior extent in the $\mathrm{S} 1$ hindlimb/forelimb region, while leaving the subplate in adjacent anterior and posterior cortical regions intact. Fluorescent microspheres coinjected with the immunotoxin are visible as a cluster of pink (in $\boldsymbol{B}$ ) or yellow (in $\boldsymbol{C}$ ) spots in the white matter near the damaged subplate. Scale bars: $\boldsymbol{B}, 1 \mathrm{~mm} ; \boldsymbol{C}, 400 \mu \mathrm{m}$. C, Bottom: Injections of anti-mouse-p75-immunotoxin ( $\mu$-toxin) do not cause SPN ablation. NeuN-stained section from a P5 rat that was injected with $\mu$-toxin at P0. Scale bar, $400 \mu \mathrm{m}$. $\boldsymbol{D}$, Left: Higher-magnification image of the ablated S1 region with damaged SPN layer from the anti-rat p75-immunotoxin-injected animal shown on the left in $\boldsymbol{C}$. Note the normal presence of neurons in the overlaying cortical plate above the ablated SPN layer. D, Middle, Unaffected S1 region with intact SPN layer from a non-injected control animal. Right, Higher-magnification image of the unaffected S1 region with intact SPN layer from the $\mu$-toxin-injected animal shown on the right in $\boldsymbol{C}$. Scale bars, $50 \mu \mathrm{m}$. $\boldsymbol{E}$, SPN removal did not influence the behavior of animals as evident from sleep-wake cycles (measured from animal motility using piezo sensors; see Materials and Methods for details).

We show that focal removal of SPNs in rats shortly after birth prevents spindle bursts, while having little effect on the occurrence of sharp potentials. The role of neuronal activity in cortical development in rodents has been investigated in the S1 barrel region (Katz and Shatz, 1996; Erzurumlu and Kind, 2001; Zhang and Poo, 2001; Feldman and Brecht, 2005; Gonzalez-Islas and Wenner, 2006; Inan and Crair, 2007). We thus investigated the effects of SPN removal in the barrel region. We find that SPN removal prevents the developmental strengthening of thalamocortical inputs to layer 4 neurons and impairs the development of the characteristic barrel pattern. Thus, SPNs are a key element in the development of the patterned organization of S1, and in the functional development of its endogenous activity patterns.

\section{Materials and Methods}

Experiments were approved by the Committee for Animal Care and Use at the University of Helsinki and the University of Maryland. We used rat pups of both sexes aged $\mathrm{P} 0$-P14 that were kept together with their parents and littermates. Postnatal day (P) 0 refers to the day of birth.

Subplate ablations. At P0-P1 (within $36 \mathrm{~h}$ after the time of birth), animals were anesthetized with isoflurane and maintained at $1-1.5 \%$ during surgery. Body temperature was kept constant using a heating pad set at $35^{\circ} \mathrm{C}$. Small craniotomies were made overlying the $\mathrm{S}$ (limb region: 0.5 posterior to $0.5 \mathrm{~mm}$ anterior from bregma; $1.7-2.3 \mathrm{~mm}$ from midline; barrel region: $0.4-0.8 \mathrm{~mm}$ posterior from bregma; $2.65-2.85 \mathrm{~mm}$ from midline) using a needle. Stereotaxic coordinates were confirmed histologically for the barrel region and electrophysiologically for the S1 limb region (see below and Results). Anti-rat p75-immunotoxin (192-IgGsaporin, MAB390, Millipore Bioscience Research Reagents; $400 \mathrm{nl}, 1 \mu \mathrm{g} /$ $\mu$ l) or control toxin (anti-mouse p75-saporin " $\mu$-toxin," or IgG-saporin "blank toxin," both from Advanced Targeting Systems; $1 \mu \mathrm{g} / \mu \mathrm{l}$ ) were dissolved in sterile physiological saline or $0.9 \% \mathrm{NaCl}$. The selectivity of p75-immunotoxin for SPNs is based on the expression of the p75 neurotrophin receptor, which is localized to SPNs in rats at P0-P1 (Fig. 1A; Koh and Loy, 1989). There are many potential caveats related to injections into neonates, thus our primary controls were anti-mouse p75-saporin and IgG-saporin injections. However, we also examined non-injected animals and vehicle-injected animals to gain insight into the potential sources of variability in the responses studied.

Toxin solutions or vehicle (physiological saline) were injected using a glass pipette (tip diameter $10-40 \mu \mathrm{m}$ ). The specificity of the p75 antibody (192-IgG) to the subplate in P0-P1 rats was confirmed by immunostaining (Fig. $1 \mathrm{~A}$ ). Fluorescent microspheres (Lumafluor Inc; 5-10\% by volume) were coinjected for visualization of injection sites. Multiple injections (2 for limb area, 3-5 for barrel area) distributed over an area within $1 \mathrm{~mm}$ were performed at $\sim 300-700 \mu \mathrm{m}$ depth. Littermates were sham-operated. After recovery in a warm $\left(35^{\circ} \mathrm{C}\right)$ environment together with a sham-operated animal, pups were placed back into their nests. All pups survived the procedure and were monitored daily for weight, feed- 
ing and general behavior. After SPN removal, freely behaving animals showed normal sleep-wake cycles similar to those in non-injected control and toxin control rats (Fig. $1 E$ ).

Injection locations were validated (Fig. 1) by the coinjected fluorescent beads, and were localized at the level of the subplate and at the edge with the underlying white matter in the $\mathrm{S} 1$ cortex hindlimb/forelimb area. Loss of SPNs was evident near the injection sites in an area of $\sim 0.75-1.5$ $\mathrm{mm}$ width rostrocaudally and mediolaterally, while no neuronal loss was observed in overlaying cortical layers and in adjacent cortical areas. Control toxin ( $\mu$-toxin, $n=4$ or blank toxin, $n=8)$ or vehicle injections $(n=$ 4 ) in the subplate did not cause observable SPN damage. Apart from the trajectory of the glass electrode used for injections or the electrode track from recordings, no further damage was observed within the cortex of toxin- or vehicle-injected animals. Anti-rat p75-toxin and control toxin injections in overlaying cortical layers that did not reach the subplate ( $n=2$ anti-rat p75-toxin, $n=4$ blank toxin) did not result in visible neuronal damage. In recordings from these animals, EEG signals appeared similar to those of non-injected controls.

A total of 149 rat pups from 57 litters were used in this study. Typically multiple animals in each litter were used for each experimental group and multiple litters were used for each experimental group. Twenty-two litters were used for EEG studies. Thirty litters were used for barrel cortex and thalamic histology. Since only one hemisphere was injected, we used the other hemisphere as within animal control. Five litters were used for in vitro physiology.

Acute head-fixed EEG recordings. EEG recordings were made between $\mathrm{P} 7$ and P10 in the S1 limb region. Surgeries were performed under isoflurane anesthesia (maintained at 1.5-2\%) using a miniature stereotaxic in which the animal was kept warm on a heating pad set at $35^{\circ} \mathrm{C}$. A window ( $\sim 1 \mathrm{~mm}$ diameter) was made overlying $\mathrm{S} 1$ guided by the location of fluorescent microspheres that were visible through the skull. Reference and ground $\mathrm{AgCl}$ electrodes $(125 \mu \mathrm{m}$, Teflon-insulated silver wire; Advent Research Materials) were placed over cerebellum. To fix the animal's head, a head post was fixed to the skull using dental acrylic. A piezo sensor (Pico sensor, TEMEC Instruments) was placed on the animal's chest to monitor breathing and other movements in parallel with the EEG (Schuchmann et al., 2006). Bipolar stimulating electrodes (75 $\mu \mathrm{m}$ insulated stainless steel with $\sim 0.5 \mathrm{~mm}$ uninsulated tips; California Fine Wire Co.) were inserted under the skin of the dorsal surface of the left fore- and hindpaw and secured with flexible collodion (Mallinckrodt Baker Inc.).

The body of the animal was wrapped in gauze and the pup was fixed into an acute recording setup, in which the pup's temperature was kept constant using a heating pad set at $37^{\circ} \mathrm{C}$. In this setting, brain temperature was found to be $32.5-33^{\circ} \mathrm{C}$ within $20-30 \mathrm{~min}$ after the surgery under isoflurane. A stable $34-35^{\circ} \mathrm{C}$ cortical temperature was found to be established within 45-60 min after surgery. Brain temperature recordings were performed in a separate group $(n=4)$ of P7-P9 rat pups using a BAT-10 digital thermometer and type T thermocouple (Physitemp Instruments), with uninsulated tip (230 $\mu \mathrm{m}$ tip diameter) that was positioned at $\sim 1-1.5 \mathrm{~mm}$ in the cortex.

Recording electrode arrays consisted of 3-6 insulated tungsten wires (20 $\mu \mathrm{m}$ diameter; California Fine Wire Co.). Electrodes were inserted into the cortex at 500-700 $\mu \mathrm{m}$ depth. To optimize event detection, additional recordings were made at deeper locations to a maximum depth of $1 \mathrm{~mm}$. The location of recordings was verified histologically and was confirmed to be at the level of middle to deep cortical layers. EEG signals were acquired at $0.16-9000 \mathrm{~Hz}$ and digitized at $32.6 \mathrm{kHz}$ (Neuralynx Digital Lynx system). Recordings started after 15-30 min recovery from anesthesia with a test session to see whether cortical EEG was detected. Recordings that were used for analysis typically started 30-60 min after the end of surgery. Spontaneous EEG activity was monitored for $30 \mathrm{~min}$ before recording evoked responses. Paws were stimulated using brief bipolar current pulses $(500 \mu \mathrm{s}, 1-10 \mathrm{~mA})$ at an interval of $30 \mathrm{~s}(10-20$ repeats). To assess the occurrence of evoked spindle bursts, paw stimulations were made at twice threshold intensity. In the present study, spindle bursts were defined spontaneous if no sensory stimulation was presented.
Chronic EEG recordings. Full-band (Fb) (DC) EEG recordings were performed as described previously (Schuchmann et al., 2006). Electrodes were implanted at P5-P6 under isoflurane (1.5-2\%). Two non-injected control rats were implanted at $\mathrm{P} 8$. Custom-made $\mathrm{AgCl}$ electrode arrays (with ca $0.5 \mathrm{~mm}$ uninsulated and chlorided tips) were inserted into middle-deep layers of the $S 1$ limb area $(0.5 \mathrm{~mm}$ posterior to $0.5 \mathrm{~mm}$ anterior from bregma; mediolateral $2.5-3.0 \mathrm{~mm}$ ) at $0.5-0.7 \mathrm{~mm}$ depth. Additional electrodes were placed outside the location of SPN ablation in the parietal (1.8-2.0 $\mathrm{mm}$ posterior from bregma; mediolateral $2.0 \mathrm{~mm}$; depth $0.5-0.7 \mathrm{~mm}$ ) and occipital cortex ( $4.0 \mathrm{~mm}$ posterior from bregma; mediolateral 2.5-3.0 mm; depth 0.5-0.7 mm). An AgCl reference and a ground electrode were placed over cerebellum (Fig. $2 A$, schematic of electrode locations). Implanted electrodes were fixed to the skull using microconnectors (Microtech Inc.) and dental acrylic. After recovery, pups were reintroduced into their litter. Starting $24-48 \mathrm{~h}$ after electrode implantation, recordings were made daily for $\sim 30$ min from P6/P7 until P10. For chronic EEG recordings, a pup was transferred from its litter to a prewarmed $\left(30-35^{\circ} \mathrm{C}\right)$ cage and placed in a small $(8 \mathrm{~cm}$ diameter $)$ recording chamber. After connection to the recording equipment and a check of the EEG signals, pups were left to acclimatize for $\sim 15 \mathrm{~min}$ before the start of recordings. In these daily sessions, pups were separated from their mother and littermates for $<1 \mathrm{~h}$. Full-band EEG was recorded using a custom-made DC amplifier (Tallgren et al., 2005). Signals were amplified $1000 \times$, and sampled at $5 \mathrm{kHz}$ using custom routines in LabVIEW (National Instruments). Breathing movements were monitored using a piezo sensor placed on the abdomen (see above). Overall motor activity of the pups was monitored by placing the recording chamber on a force displacement transducer (Grass Technologies) and by video (Philips SPC900NC). The force transducer sensitivity was high enough to detect small movements such as isolated sleep twitches and breathing patterns. For both acute and chronic recordings, electrode location was confirmed to be in the middle or deep cortical layers (Fig. $2 A$ ).

EEG analysis. Off-line analysis was done using Diadem, LabVIEW and MATLAB. For characterization of the spontaneous EEG at least 20 min of sleeping time was analyzed for each day of recording per animal. For chronic recordings events were analyzed from 1 day or from 2 consecutive days of recording between P7 and P10, from which the mean frequency of occurrence was calculated. Waking periods were excluded from analysis to minimize contamination of signals by movement artifacts on the EEG. Detection of movement artifacts was done by comparison of EEG traces with the video and movement recordings. Spindle bursts were identified by eye and confirmed after filtering the raw EEG using a $5-40 \mathrm{~Hz}$ bandpass-filter (Butterworth 4 th order), in agreement with described S1 spindle burst criteria for neonatal rats in vivo (Khazipov et al., 2004). Only oscillatory events lasting $>100 \mathrm{~ms}$ and containing at least 4 separate peaks with amplitudes larger than $25 \mu \mathrm{V}$ were considered for analysis. Identification of spindle bursts was further guided by inspection of their time-frequency (TF) characteristics (Minlebaev et al., 2007). The duration of spontaneous spindle burst activity was analyzed from the $5-40 \mathrm{~Hz}$ bandpass filtered EEG, taking into account only oscillations exceeding $25 \mu \mathrm{V}$ in amplitude. Calculation of TF characteristics of spontaneous spindle burst activity was done by Morlet-wavelet-based TF analysis using custom-written functions in LabVIEW. The spindle peak frequency of the nested oscillations in the $5-45 \mathrm{~Hz}$ range was taken from the wavelet amplitude spectrum obtained as an average across a $2 \mathrm{~s}$ window with a bank of 13 Morlet wavelets with log-linearly distributed center frequencies and a width parameter $\mathrm{m}=5$.

Neocortical sharp potentials were recognized as single sharp events of relatively short duration (Bernard et al., 2005). Only sharp potentials with amplitudes larger than $25 \mu \mathrm{V}$ were analyzed. Cumulative root-mean-square (cRMS; Ruusuvuori et al., 2004) values of spontaneous S1 EEG were quantified for the frequency bands of $1-4 \mathrm{~Hz}, 4-10 \mathrm{~Hz}$, or 5-45 Hz from EEGepochs of 15-20 min in duration. For comparison between control groups and ablated animals, values were calculated as cRMS/s and presented as percentage of the mean value of non-injected controls.

The power and peak frequency of evoked spindle burst activity was calculated by fast Fourier transform over a $500 \mathrm{~ms}$ window from the 5-40 $\mathrm{Hz}$ bandpass filtered (second order Butterworth) local field potential (LFP) response. 

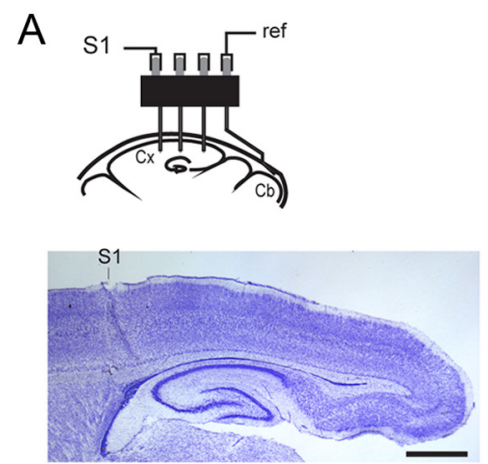

C non-injected control

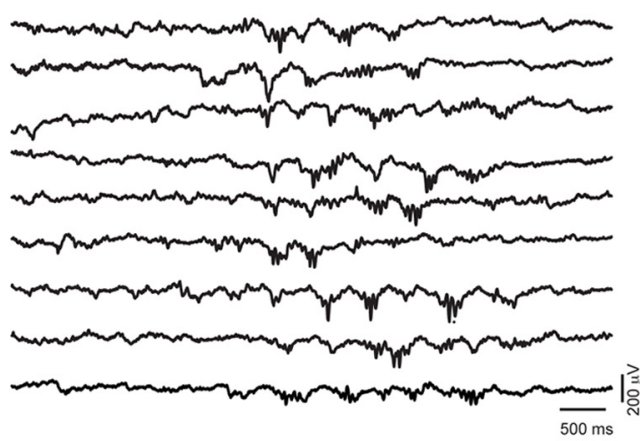

E

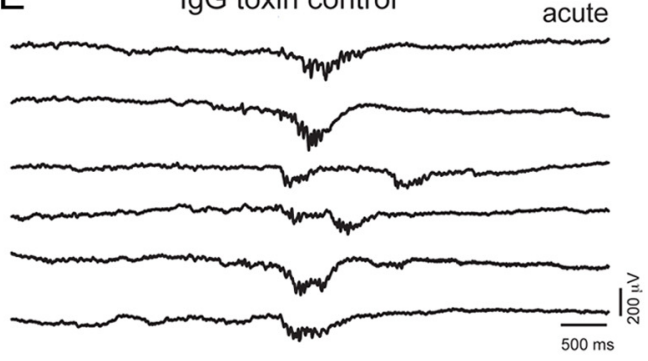

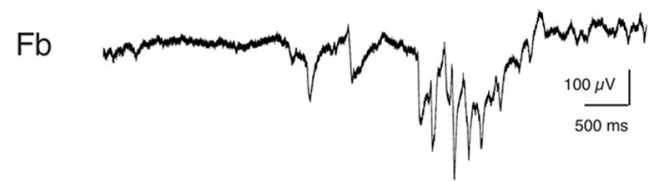

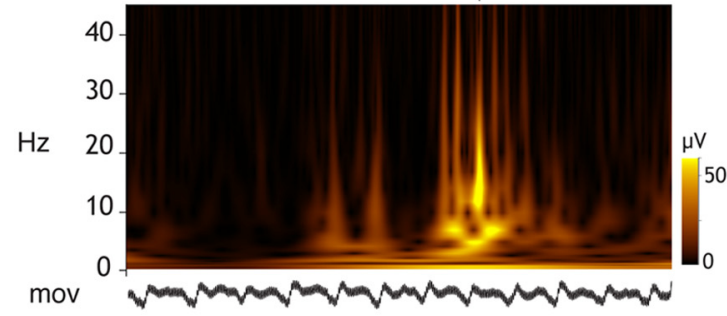
D
ablated (-SPN)
chronic
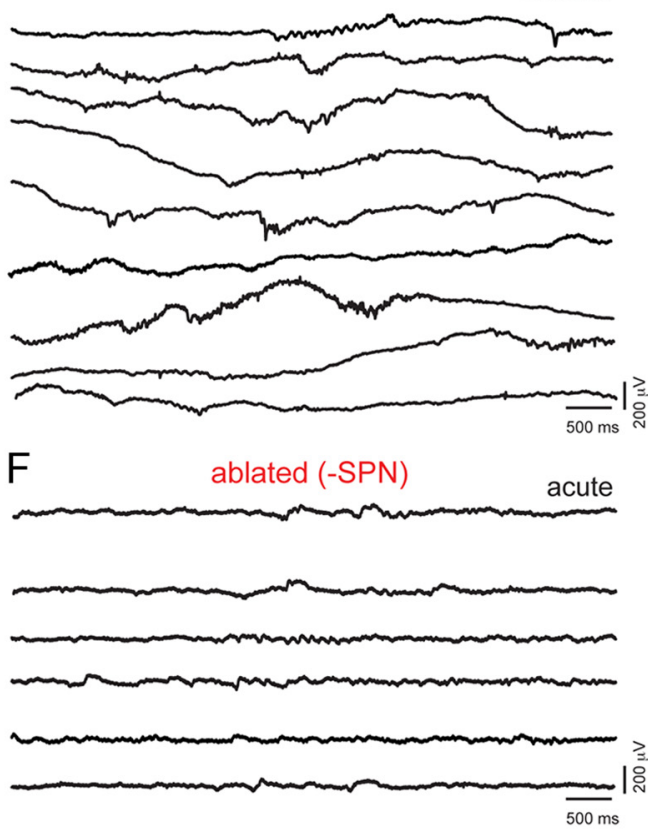

G Occurrence $\mathrm{H}$ Peak freq. I Duration J cRMS $10-45 \mathrm{~Hz}$ K

Occurrence
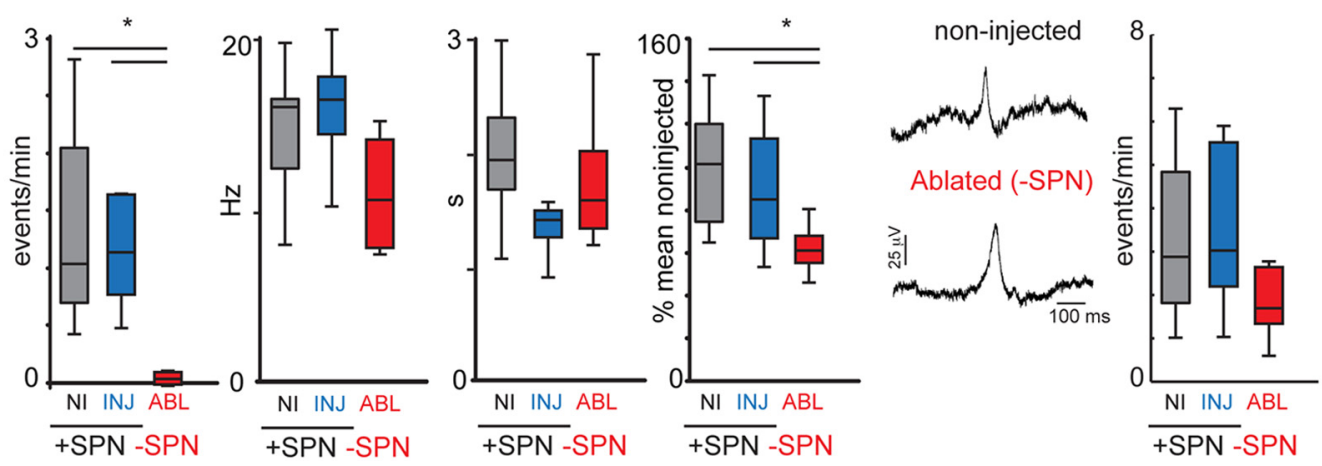

Figure 2. SPN are required for the generation of spindle bursts in immature S1 limb region. $A$, Scheme depicting the electrode configuration used for chronic EEG recordings. Photomicrograph shows the location of the $\mathrm{AgCl}$ electrode track in deep cortical layers of the $\mathrm{S} 1$ limb region of a P9 non-injected control rat. Scale bar, $1 \mathrm{~mm} . \boldsymbol{B}$, Sample trace from a P8 animal showing the frequency composition of a spindle burst. Top trace shows Fb-EEG, in the middle is the time-frequency plot showing the frequency composition. The lower trace (labeled "mov") shows the signal from the movement sensor, indicating regular breathing. Note that spindle bursts can occur in the absence of limb and trunk movements. $C-F$, Segments of EEG traces under various conditions. Segments containing spindle bursts were aligned to facilitate visual inspection. C, Spindle burst activity is observed regularly in the S1 hindlimb/forelimb region of rats between P7 and P10. Multiple traces showing spontaneous spindle burst activity from a freely behaving non-injected control rat at P10. D, After SPN ablation at P0 -P1, spindle burst activity is largely absent and the figure shows the most salient events during the recording epoch. Example traces from a freely behaving P7 rat with local SPN ablation in S1. Sharp potentials are less affected and are regularly seen at a bit reduced frequency compared with injected control animals (see Fig $1 \mathrm{~K}$ for details). $E$, Control toxin injections (lgG toxin) at P0-P1 do not impair the expression of spindle burst activity at P7-P10. Example traces from a head-fixed $\mathrm{P9}$ rat showing regular spindle burst activity recorded in the $S 1$ hindlimb area. $F$, EEG recorded from the $S 1$ hindlimb region of a head-fixed $S P N$-ablated rat at $P 9$, showing faint oscillations, most of which did not meet the spindle burst criterion with prominent activity in the $5-40 \mathrm{~Hz}$ range (see Materials and Methods). G-I, Box plots summarizing occurrence of spindle bursts $(\boldsymbol{G})$, spindle burst peak frequency $(\boldsymbol{H})$, and spindle burst duration $(\boldsymbol{I}) .{ }^{*} p<0.05$, significant reduction in spindle burst occurrence after ablation. Two of the three chronically recorded ablated rats did not show any spindle burst activity in the recording sessions, performed at P7 and P8. Box plots show the median \pm quartile values from the pooled (Figure legend continues.) 
In vitro electrophysiology. Rat pups were deeply anesthetized with isoflurane (Halocarbon). A block of brain containing S1 and the thalamus was removed and thalamocortical slices $(500 \mu \mathrm{m}$ thick; (Agmon and Connors, 1991) were cut on a vibrating microtome (Leica) in ice-cold physiological solution containing the following (in $\mathrm{mm}$ ): $130 \mathrm{NaCl}, 3$ $\mathrm{KCl}, 1.25 \mathrm{KH}_{2} \mathrm{PO}_{4}, 20 \mathrm{NaHCO}_{3}, 10$ glucose, $1.3 \mathrm{MgSO}_{4}, 2.5 \mathrm{CaCl}_{2}(\mathrm{pH}$ $7.35-7.4$, in $\left.95 \% \mathrm{O}_{2}-5 \% \mathrm{CO}_{2}\right)$. Slices were incubated for $1 \mathrm{~h}$ at $30^{\circ} \mathrm{C}$ and then kept at room temperature. For recording, slices were held in a chamber on a fixed stage microscope (Nikon FN1) and superfused (2-4 ml/ min) with $127 \mathrm{NaCl}, 3 \mathrm{KCl}, 1.25 \mathrm{KH}_{2} \mathrm{PO}_{4}, 20 \mathrm{NaHCO}_{3}, 10$ glucose, 4.5 $\mathrm{MgSO}_{4}, 2.5 \mathrm{CaCl}_{2}\left(\mathrm{pH} 7.35-7.4\right.$, in $95 \% \mathrm{O}_{2}-5 \% \mathrm{CO}_{2}$ ) at room temperature to reduce spontaneous activity in the slice. The location of the recording site in S1 was identified by appearance of barrels under DIC. Whole-cell recordings were performed with a patch-clamp amplifier (Multiclamp 700B, Molecular Devices) using pipettes with input resistance of 4-8 M $\Omega$. Data were acquired with a Digidata AD board (Molecular Devices) under pClamp (v9 and v10) and analyzed offline using MATLAB (MathWorks). Electrodes were filled with (in $\mathrm{mm}$ ) 110 K-gluconate, $4 \mathrm{KCl}, 4 \mathrm{NaCl}, 0.2 \mathrm{CaCl}_{2}, 10 \mathrm{HEPES}, 1.1 \mathrm{EGTA}, 2 \mathrm{Mg}$-ATP, $1 \mathrm{MgCl}_{2}$ and 5 glutathione ( $\mathrm{pH} 7.2,300 \mathrm{mOsm}$ ) for spiking patterns or with (in mM) 115 cesium methanesulfonate $\left(\mathrm{CsCH}_{3} \mathrm{SO}_{3}\right), 5 \mathrm{NaF}, 10$ EGTA, 10 HEPES, 15 CsCl, 3.5 MgATP, 3 QX-314 (pH 7.25, 300 mOsm) for EPSC measurements. Biocytin or Neurobiotin (0.5\%) was added to the electrode solution as needed. Membrane voltages were corrected for an estimated liquid junction potential of 14 or $10 \mathrm{mV}$ respectively. For electrical stimulation we use a bipolar electrode (Microprobe, biphasic, $0.2 \mathrm{~ms}$, at $0.03 \mathrm{~Hz}$ ) coupled to a stimulus isolator (Cygnus). The electrode was placed into the thalamocortical projections at a position that maximized the EPSC for each cell.

Immunohistochemistry. p75 and NeuN staining was performed using techniques described previously (Tolner et al., 2003) with mouse anti-rat p75 antibody 192 IgG (MAB365, Millipore Bioscience Research Reagents; 1:200) or mouse anti-rat NeuN antibody (MAB377, Millipore Bioscience Research Reagents; 1:1000).

Cytochrome oxidase staining and barrel analysis. Rat pups were perfused as described above and the two hemispheres were postfixed for $2 \mathrm{~h}$ between 2 glass coverslips (spaced $1500 \mu \mathrm{m}$ ) to flatten the cortex. After cryoprotection $50 \mu \mathrm{m}$ horizontal slices were cut on a freezing microtome or cryostat spanning the entire range of the cortex. Slices were incubated at $37^{\circ} \mathrm{C}$ with $20 \mathrm{mg}$ of $\mathrm{DAB}, 25.0 \mathrm{mg}$ of cytochrome $c$ (from horse heart, Sigma-Aldrich), $2.0 \mathrm{~g}$ of solid sucrose in $50.0 \mathrm{ml}$ of PBS for 30-45 min, washed once in PBS, dehydrated, mounted and coverslipped. Pictures were taken from multiple sections (up to 6) covering the entire depth extent of the barrel field. The presence of each barrel in each hemisphere was confirmed by aligning and merging the images using Photoshop (CS5, Adobe; see Fig. 5). The location of the injection site was determined from fluorescent beads contained in sections through the white matter or from evidence of the pipette track. Due to the number of washes and incubation steps, beads were not detectable in all sections. The presence of the full barrel pattern in different animals was performed qualitatively by judging on a scale from 0 to 4 the presence of a complete barrel field. Scores of 0 indicated the presence of complete barrel field while scores of 1-4 indicated small (1, indicating a change in barrel shape), mild (2, indicating some missing barrels), medium (3, indicating missing rows or columns), or large (4, missing large parts) disruptions of the barrel field respectively. For judgment we used 4 independent observers blind to manipulation and animal ID. The scores of the observers for each animal were averaged.

$\leftarrow$

(Figure legend continued.) acute and chronic values from non-injected controls ( $n=10$ for $\mathrm{G}$; $n=9$ for $\mathrm{H}$ ), control-injected controls $(n=13)$ and ablated rats $(n=7)$. J, Box plot shows cRMS values for the $10-45 \mathrm{~Hz}$ frequency range in spontaneous EEG recordings. ${ }^{*} p<0.05$, significant reduction in cRMS after ablation. $\boldsymbol{K}$, Left, Sample EEG traces of sharp potentials from a freely behaving (chronic) non-injected control animal with $(+S P N)$ or an ablated animal without SPN (-SPN). Right, Sharp potential frequency (events/min) in the S1 limb region during periods of immobility from freely behaving (chronic) and head-fixed (acute) recordings, in P7-P10 rats with intact $(+)$ and locally ablated $(-)$ SPN.
Statistics. Statistical analysis between groups was performed using the Mann-Whitney $U$ test or Student's $t$ test, with two-tailed distribution. Average values are expressed as mean \pm SE unless stated. Statistical significance was taken at $p<0.05$.

\section{Results}

\section{Local removal of subplate neurons in neonatal rats using} p75-immunotoxin

Expression of the p75 neurotrophin receptor is localized to SPNs in rats at P0-P1 (see also Koh and Loy, 1989). We used immunotoxins against p75 neurotrophin receptor-expressing cells to selectively remove SPNs (Kanold et al., 2003; Kanold and Shatz, 2006). We confirmed the specificity of the p75 antibody used for SPN ablations in rat by immunostaining (Fig. $1 A$ ). Since the neurodegenerative effect of p75-immunotoxin has been reported to become evident histologically after $\sim 2-4 \mathrm{~d}$ (Book et al., 1995), and since EEG recordings (see below) are technically difficult to carry out in very young rat pups due to their weak skull, we studied the effects of SPN damage in rats $\sim 1$ week after the immunotoxin injections. The localized immunotoxin injections in the subplate (barrel or limb region) caused loss of SPNs near the injections in an area of $\sim 0.75-1.5$ $\mathrm{mm}$ width rostrocaudally and mediolaterally while leaving the overlying cortical plate intact (Fig. $1 C, D ; n=7$ ablated rats, limb region). Injections of control toxin or vehicle into the subplate did not cause loss of SPNs (Fig. $1 C, D ; n=4 \mu$-toxin, $n=8$ blank toxin, $n=4$ vehicle-injected rats, limb region). Behaviorally, animals with local SPN ablation were indistinguishable from control non-injected, vehicle-injected or control toxin-injected rats and showed normal sleep-wake cycles as judged by their mobility patterns (Fig. $1 E$ ).

\section{Subplate removal prevents spontaneous spindle bursts in the S1 limb region}

To identify the functional consequences of SPN removal on spindle bursts, we used chronic Fb-EEG recordings (labeled "chronic" in Fig. 2) from freely moving P7-P10 rats (Sipilä et al., 2006) to investigate spindle bursts (Fig. 2A,C,D). Because spindle bursts have been well characterized in the $S 1$ limb region (Khazipov et al., 2004; Yang et al., 2009) and since the limb allows selective sensory stimulation we recorded EEG activity from the $\mathrm{S} 1$ limb region. We used Fb-EEG, since the low-frequency component of spindle bursts in rats is attenuated and distorted by high-pass filtering in standard AC recordings (Minlebaev et al., 2009). Since it is technically challenging (and possibly damaging) to chronically implant electrodes in rat pups younger than P5, especially considering the subplate injections at $\mathrm{P} 0-\mathrm{P} 1$, electrodes were implanted in subplate-targeted pups at P6, after which we studied the functional effect of subplate ablation. In the chronic freely moving recordings, pups were recorded between P7 and P10 in daily sessions of $\sim 30-45$ min in a small prewarmed recording chamber. Movements (including breathing) were monitored by a force transducer coupled to the recording platform as well as by a piezo element connected to the pup's body and video (see Materials and Methods for details). To constrain potential movement artifacts we recorded in a second cohort of animals using acute head-fixed preparations (labeled "acute" in Fig. 2). We did not see any effects of head fixation on spindle burst activity; hence the acute and chronic EEG data were pooled.

Spindle bursts consisted of higher frequencies in the $\alpha$ to $\gamma$-range embedded in a $\delta$ wave (Fig. $2 B$; Marcano-Reik and Blumberg, 2008; Minlebaev et al., 2009; Mohns and Blumberg, 
2010). Under control conditions ( $n=12$ non-injected, $n=8$ blank toxin, $n=2 \mu$-toxin, $n=3$ vehicle-injected rats), spindle bursts were observed regularly in acute or chronic recordings, typically occurring at a rate of $\sim 0.5-3$ events/min (Fig. $2 C, E, G$ ). Since chronic and acute experiments showed that injection per se did not influence SB occurrence, the data for blank toxin, $\mu$-toxin and vehicle-injected controls were pooled and will be referred to as "control-injected." In contrast to the control-injected animals, following SPN removal spindle bursts were rarely observed (Fig. $2 D, F)$. The rate of occurrence following subplate ablation was $0.09 \pm 0.04$ events $/ \mathrm{min}(n=7)$ versus $1.48 \pm 0.31$ events $/ \mathrm{min}$ in control-injected animals $(n=13 ; p<0.0001$; Fig. $2 G)$. The peak frequency of the spindle bursts in ablated rats $(11.1 \pm 1.5 \mathrm{~Hz} ; n=$ 1 of 3 chronically recorded pups; $n=4$ of 4 acutely recorded pups) was slightly reduced compared with that of controlinjected pups $15.5 \pm 0.8 \mathrm{~Hz} ; p=0.035)$ but within the range of $8-20 \mathrm{~Hz}$ observed for non-injected animals (Fig. $2 \mathrm{H}$ ). The duration of remaining spindle bursts in ablated rats $(1.77 \pm 0.24 \mathrm{~s} ; n=$ 1 of 3 chronically recorded pups; $n=4$ of 4 acutely recorded pups) was comparable to that of control-injected pups (1.60 \pm $0.15 ; n=13 ; p=0.6)$ or non-injected animals ( $1.77 \pm 0.29 \mathrm{~s}, n=$ 10; Fig. $2 I)$.

The cRMS of the EEG is an unbiased measure of total activity. A high rate of occurrence of spindle bursts leads to a high cRMS in the appropriate frequency band, while a reduction in the rate of spindle bursts has the opposite effect. Thus we investigated whether the loss of spindle bursts in the $\mathrm{S} 1 \mathrm{limb}$ region is reflected in the cRMS of the EEG. We first measured the cRMS in the frequency band that comprises most spindle burst activity. The cRMS in the $10-45 \mathrm{~Hz}$ band was reduced after ablation but not in control-injected animals $(62.5 \pm 4.2 \%$ for ablated, $n=7$ versus $103.2 \pm 13.6 \%$ for control-injected rats $n=13$; $p=0.013$; calculated as percentage of the mean value for non-injected controls; Fig. $2 J)$. In addition, cRMS values for the $1-4 \mathrm{~Hz}$ and $4-10$ $\mathrm{Hz}$ frequency bands - in which spindle bursts also contain energy-were also lower in ablated than in control-injected rats ( $[1-4 \mathrm{~Hz}]: 57.9 \pm 8.8 \%$ for ablated versus $95.9 \pm 12.7 \%$ controlinjected, $p=0.0449$; $[4-10 \mathrm{~Hz}]: 58.8 \pm 8.6 \%$ for ablated vs $107.2 \pm 18.2 \%$, control-injected; $p=0.0556)$. In some ablated animals we observed conspicuous large amplitude DC shifts (up to $500 \mu \mathrm{V}$ ) of several seconds duration and of unknown origin (Fig. 2D).

In addition to spontaneous spindle bursts, sharp potentials are a second activity pattern that is regularly observed in neonatal rat cortex (Khazipov et al., 2004; Bernard et al., 2005; Seelke and Blumberg, 2010). We therefore investigated whether the presence of sharp potentials in the S1 limb region is affected by local subplate removal. In non-injected and controlinjected animals, sharp potentials were present at rates that ranged between 1 and 6 events per minute $(3.6 \pm 0.5$ events/ $\min , n=13$ control-injected animals; Fig. $2 \mathrm{~K}$ ). After SPN ablation, sharp potentials were still present $(1.8 \pm 0.3$ events/ $\min , n=7$ ablated rats; $p=0.056$, Mann-Whitney; Fig. $2 K$ ). In chronic EEG recordings in freely moving rats, additional recordings from parietal and occipital regions also showed regular sharp potentials in ablated rats $(n=3)$ as seen for animals in the control groups $(n=6$ non-injected; $n=2$ $\mu$-toxin and $n=2$ blank toxin; data not shown).

Thus, while SPNs are required for the generation of spontaneous spindle bursts they do not seem to play a role in the modulation of the frequency of cortical sharp potentials.

\section{Subplate removal prevents sensory evoked spindle bursts in the $S 1$ limb region}

Evoked activity in the immature S1 is known to uniformly consist of spindle bursts (Khazipov et al., 2004; Minlebaev et al., 2007; Yang et al., 2009). To reliably activate all afferent projections, we electrically stimulated the forepaw or hindpaw. Electrical stimulation of the forepaw or hindpaw led to evoked spindle bursts in $\mathrm{S} 1$ in non-injected, vehicle-injected, and control-toxin-injected animals (Fig. $3 A$ ). In ablated rats, evoked activity was markedly reduced and contained much less activity in the spindle burst range (Fig. $3 B$ ). This reduction in spindle burst activity in single trials was also visible in the average LFP (Fig. $3 C$ ). We quantified the impairment in evoked $\mathrm{S} 1$ activity by calculating the ratio of stimulation trials in which responses contained spindle burst activity, which was reduced by more than half for the ablated animals $(0.30 \pm 0.06, n=4$ ablated vs $0.67 \pm 0.08, n=8$ controlinjected animals; $p=0.0108$; Fig. $3 D$ ). This indicates a reduction in the stimulus response relation in S1 following subplate removal. To further characterize the evoked responses, we computed the power in the $5-40 \mathrm{~Hz}$ "spindle" frequency range of EEG in the first $500 \mathrm{~ms}$ following the stimulation. The power was reduced in ablated animals $\left(6760 \pm 3019(\mu \mathrm{V})^{2} / \mathrm{ms}, n=4\right)$ compared with control-injected animals $\left(22,157 \pm 3775(\mu \mathrm{V})^{2} /\right.$ $\mathrm{ms}, n=8 ; p=0.0162$; Fig. $3 E$ ). Thus, together these data suggest that SPNs are important not only for the generation of spontaneous spindle bursts but also for the development of early sensory evoked responses.

\section{Subplate removal prevents strengthening of thalamocortical connections}

Our in vivo recordings showed a marked reduction in both spontaneous and sensory evoked activity in the S1 limb region. The reduced activity can arise from abnormal firing of cortical neurons or from altered synaptic connectivity, especially of thalamocortical synapses. Thalamocortical connectivity and patterning can be probed in vitro in the S1 barrel region (Katz and Shatz, 1996; Erzurumlu and Kind, 2001; Zhang and Poo, 2001; Feldman and Brecht, 2005; Gonzalez-Islas and Wenner, 2006; Inan and Crair, 2007). To investigate these possibilities we ablated SPNs in the $S 1$ barrel region and $\sim 10 \mathrm{~d}$ after subplate removal performed whole patch-clamp recordings from layer 4 neurons $(n=106)$ in slices of S1 ( $n=30$ animals). We first investigated how SPN removal affects the intrinsic properties of cells in layer 4, the target layer of SPN projections (Zhao et al., 2009). Current injections in cells in control and ablated areas readily evoked action potentials in both sets of neurons ( $n=15$ and $n=20$ cells respectively; Fig. 4A). By injecting currents of different amplitudes we found that input resistance and resting potential of layer 4 neurons in control and ablated areas were similar (both $p>$ 0.05 ; Fig. $4 B$ ). These results suggest that removal of SPNs in rat pups does not grossly affect the intrinsic properties of cortical neurons. Since SPNs are implicated in the maturation of thalamocortical synapses in cat visual cortex (Kanold et al., 2003) we next investigated whether these neurons play a similar role in rodent $\mathrm{S} 1$. We thus stimulated thalamocortical projections while recording from layer 4 neurons (Fig. $4 C$ ). In both populations of neurons, short latency EPSCs were observed (Fig. 4D, left). Increasing the stimulation level led to an increase in EPSC amplitude (Fig. $4 D$, right, showing example responses of two cells). We found that the maximum EPSC amplitude was significantly smaller for layer 4 cells from ablated animals ( $n=26$ cells) than in layer 4 cells from non-injected $(n=17)$, vehicle $(n=14)$, or control-toxin $(n=14)$-injected animals (Fig. $4 E$, $p<0.0001)$. 
These results show that thalamocortical transmission is impaired after subplate removal.

\section{Subplate removal prevents barrel patterning}

An organizational hallmark of S1 are the whisker barrels where thalamic afferents representing the whiskers form a barrel pattern (Katz and Shatz, 1996; Erzurumlu and Kind, 2001; Zhang and Poo, 2001; Feldman and Brecht, 2005; Gonzalez-Islas and Wenner, 2006; Inan and Crair, 2007). The formation of this pattern has been shown to depend on glutamatergic activity (Fox et al., 1996; Iwasato et al., 2000). Spindle bursts have been suggested to be instructive in both the functional and structural development of barrel cortex (Minlebaev et al., 2007). In light of the disruption of spindle bursts following subplate removal we therefore hypothesized that the functional organization of S1 might be impaired. We thus tested whether this organization depends on SPNs. We ablated SPNs at P0 and performed cytochrome oxidase staining $\sim 10$ $\mathrm{d}$ after subplate removal and quantified the presence of all barrels in the S1 whisker field. In non-injected $(n=7)$, controltoxin-injected $(n=19)$, and vehicleinjected hemispheres $(n=5)$, we observed a normal pattern characterized by the presence of densely stained barrels in each row and position (Fig. $5 A, C$; each $p>0.1$ compared with non-injected control). In contrast, in hemispheres where SPNs had been removed the barrel pattern showed large gaps or complete absence of densely stained barrels depending on the size of the injected area $(n=15$, compared with control-toxin-injected animals $p=$ 0.0012; compared with non-injected $p=$ 0.0168; Mann-Whitney; Fig. 5A-C). In animals in which fluorescent beads were recovered, the gaps in the barrel pattern were located superficial to the location of the beads in the deeper sections (Fig. 5B). The variability of the size of the gaps might be due to targeting variability and/or variability in the maturity of barrel cortex at the time of ablation (Fig. $5 C$ ). The effects of SPN removal were restricted to the cortex as barreloids in the thalamus were present after subplate removal $(n=4$ animals, Fig. $5 D)$. Thus SPNs are required for the normal patterning of S1 barrel cortex in rodents. Together our data show that SPNs are required for normal activity patterns and for the development of functional organization of rodent $\mathrm{S} 1$.

\section{Discussion}

Our results show that the presence of SPNs in the newborn rat cortex is required for the generation of spindle bursts, a specific type of self-organized activity in the immature cortex (Khazipov et al., 2004). Consistent with our finding that removal of SPNs controls (+SPN)
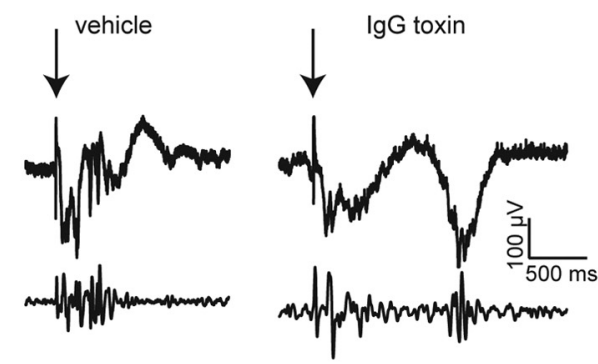

C
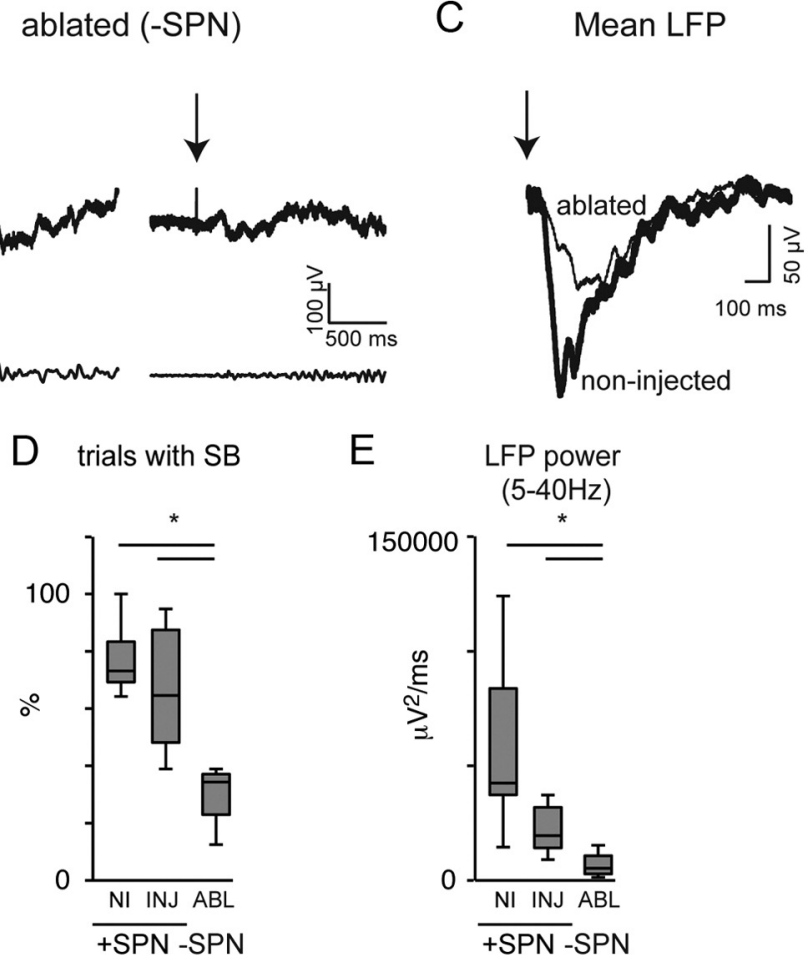

E LFP power

$(5-40 \mathrm{~Hz})$

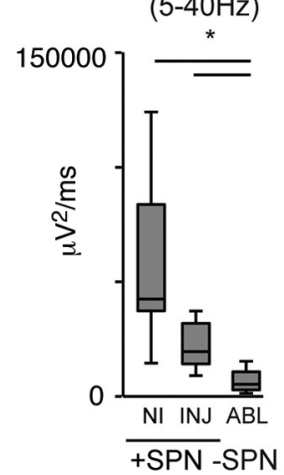

Figure 3. SPNs are required for the expression of sensory evoked spindle burst activity in the rat $S 1$ limb region. $A$, Single-trial LFP responses in middle-deep layers of the $S 1$ limb region to electrical stimulation of the hind- or forepaw for control animals (non-injected, vehicle-injected, or control lgG toxin-injected; +SPN). The 5-40 Hz bandpass filtered traces reveal evoked spindle burst activity. $\boldsymbol{B}$, Single-trial LFP responses to electrical stimulation of the hind- or forepaw in subplate-ablated animals (-SPN). After local SPN ablation, evoked spindle burst activity in the $S 1$ hindlimb/forelimb region is significantly reduced. $C$, Mean evoked LFP of a non-injected and ablated animal illustrates the lower amplitude of the LFP after local SPN ablation. $\boldsymbol{D}$, The ratio of evoked responses with spindle burst activity (see Materials and Methods) is reduced in rats with local SPN ablation. ${ }^{*} p=0.0108$ for comparison of control-injected ( $n=8$ ) with ablated rats $(n=4$; two-tailed Mann-Whitney $U$ test). $\boldsymbol{E}$, Evoked LFP power in the $5-40 \mathrm{~Hz}$ band is reduced for ablated animals $\left(n=4 ;{ }^{*} p=0.0162\right.$ for comparison with injected controls $[n=8]$; two-tailed Mann-Whitney $U$ test). Shown are responses to stimulation at $2 \times$ threshold intensity. Threshold stimulation levels were higher in ablated than in toxin control animals ( $2.0 \mathrm{~mA}$ for ablated rats vs $0.7 \pm 0.2 \mathrm{~mA}$ for injected controls).

reduces spindle burst activity we find that thalamocortical transmission is impaired. In addition we observed a disruption of the development of the patterning of the barrel field. Prior work has implicated spindle bursts in the formation of barrels (Minlebaev et al., 2007). Although our data do not conclusively show a causal link between spindle burst activity and barrel formation, our observations are consistent with the idea that spindle bursts promote the development of thalamocortical organization.

What is the role of SPNs in the generation of spindle bursts? While spindle bursts can be triggered by peripheral sensory inputs (Khazipov et al., 2004; Marcano-Reik and Blumberg, 2008; Minlebaev et al., 2009; Colonnese et al., 2010; Mohns and Blumberg, 2010), they can also occur in the absence of such inputs (Khazipov et al., 2004; Hanganu et al., 2006). Therefore, while 
A

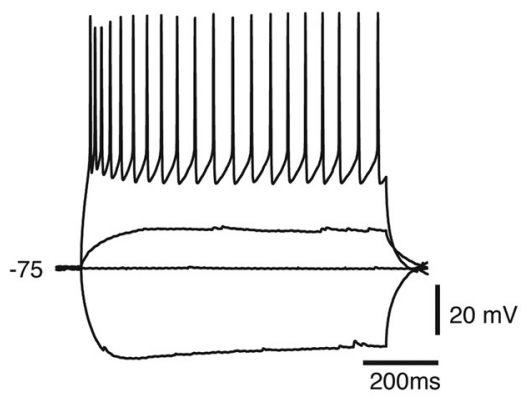

B

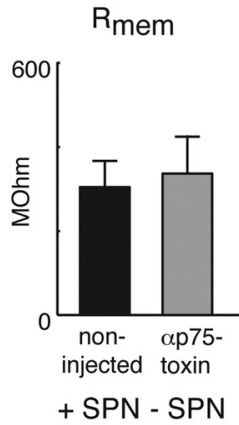

$V_{\text {rest }}$

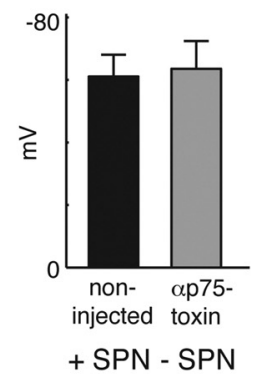

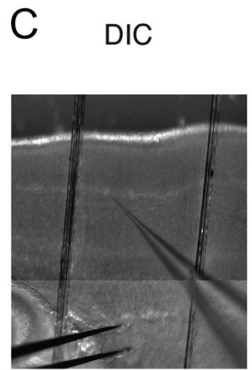

D

non-injected
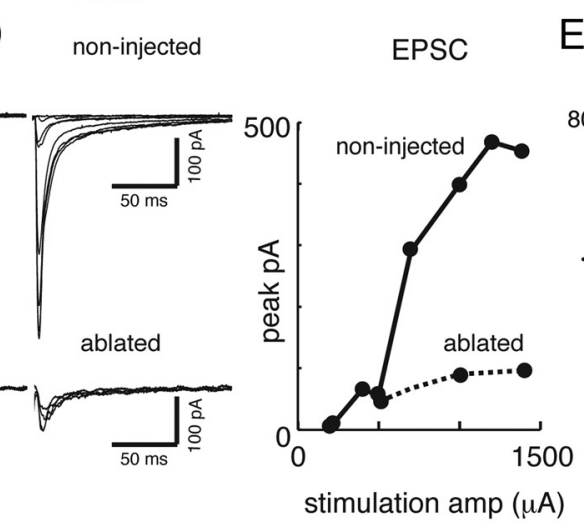

stimulation amp $(\mu \mathrm{A})$
E

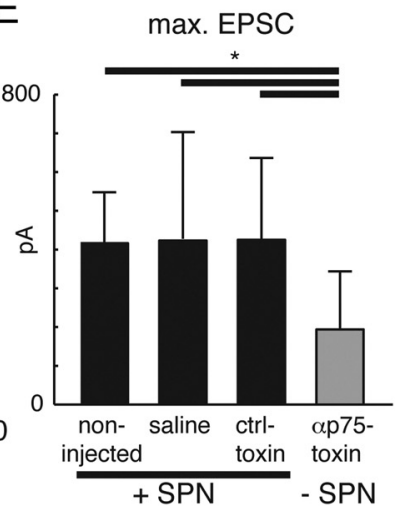

Figure 4. SPN ablation in the S1 barrel cortex in rats alters thalamocortical synaptic strength in layer 4 neurons. $A$, Whole-cell recording of layer 4 principal neuron in a thalamocortical slice from a subplate-ablated P10 rat. Traces show responses to multiple current injections. $\boldsymbol{B}$, Neurons from control vs ablated slices have similar input resistance $(304.4 \pm 62 \mathrm{M} \Omega$ vs $336.6 \pm 89.9 \mathrm{M} \Omega$; mean $\pm S D, p>0.1)$ and resting potential $(-61.1 \pm 6.9 \mathrm{mV}$ vs $-64.4 \pm 8.3 \mathrm{mV}$; mean $\pm S D, p>0.1)$. Recordings were made in slices from P10 rats, after local SPN targeting at P0 for ablated or control-injected animals. C, Recording configuration showing the recording pipette in layer 4 and stimulation electrode in the thalamocortical projections. D, Left, Traces show evoked EPSCs of a representative neuron from each population to stimulations with varying stimulation intensities. Right, Graph shows EPSC amplitude for the two neurons shown on the left as a function of stimulation strength. Note that the maximum EPSC is smaller in layer 4 cells from the SPN-ablated region. E, Shown are the maximal EPSCs for all recorded SPNs in each group. The maximum evoked EPSC amplitude is $\sim 50 \%$ smaller in slices from SPN-ablated animals than in slices from non-injected, control-toxin-injected, or saline-injected animals $(194.1 \pm 148.8 \mathrm{pA}$ vs $415.3 \pm 127.6 \mathrm{pA}$, $420.9 \pm 212.8 \mathrm{pA}, 421.7 \pm 276.9 \mathrm{pA}$; mean $\left.\pm S D,{ }^{*} p<0.0001 ; A N O V A\right)$. Evoked EPSC amplitudes from slices of saline- and control-toxin-injected animals are indistinguishable from control ( $p>$ 0.1 ; ANOVA).

they can be triggered by external stimuli, the generation of spindle burst activity seems to rely to a large extent on circuits intrinsic to the CNS. Thus, what circuits underlie the generation of spindle bursts? The thalamus was shown to be active during spindle burst activity in vivo at earlier ages and has been suggested to actively contribute to spindle burst generation (Khazipov et al., 2004; Evrard and Ropert, 2009). Thalamic activity is relayed to the cortex. SPNs are located between thalamus and the thalamorecipient layer 4, receive thalamic inputs, and provide excitatory inputs to layer 4 (Friauf and Shatz, 1991; Hanganu et al., 2002; Zhao et al., 2009). Thus, SPNs receive and relay thalamic inputs to layer 4. Therefore SPNs can mediate spindle burst activity. In line with such a role, we observed impaired sensory-evoked responses including abolishment of spindle bursts as well as impaired thalamocortical transmission in the absence of SPNs. These results mirror previous results in cat that were obtained after much longer post-ablation periods (Kanold et al., 2003) and show strong short-term effects of SPN removal. SPNs might play multiple roles in mediating spindle burst activity. The absence of both spontaneous and evoked cortical spindle burst activity after loss of SPNs could be a direct result of the missing relay in the subplate and/or of impaired maturation of thalamocortical connectivity. In this case SPNs might play a permissive role in promoting spindle bursts. In addition, SPNs might be directly involved in the generation of spindle bursts since they are active during these events (Yang et al., 2009) and tend to oscillate in the spindle-frequency range (Hirsch and Luhmann, 2008).
Since thalamocortical transmission is impaired in the absence of SPNs and since we still observed a large number of sharp potentials consequent to SPN removal, it is likely that circuits intrinsic to the cerebral cortex can generate sharp potentials. In agreement with this, in vitro work has shown that sharp potentials but not spindle bursts can be recorded in neocortical slices from immature mice, which lack thalamocortical circuits (Rheims et al., 2008).

The low level of remaining spindle burst activity that was observed in the ablated $S 1$ region could originate from nearby unaffected regions of S1. Although spindle burst activity in vivo has been demonstrated to be spatially confined, spindle bursts were shown to slowly spread within an area of at least a couple of $\mathrm{mm}$ across S1 (Khazipov et al., 2004). It was demonstrated in vitro that the intracolumnar synchronization of subplate-driven cholinergic oscillations in neonatal S1 is mediated by gap junctions (Dupont et al., 2006), and it has been indicated by other in vitro work that a similar mechanism could underlie the slow spread of early network activity across S1 (Sun and Luhmann, 2007).

Although substantial changes in spindle burst rate have been reported to occur toward the end of the first postnatal week in rats (Marcano-Reik et al., 2010), SPN ablation caused comparable low rates of spindle burst occurrence for the different animals in both acute and chronic recordings within the P7-P10 time window studied. A fall in brain temperature below the physiological level of $37^{\circ} \mathrm{C}$ is known to have strong effects on network activity. The brain temperature of $\sim 34-35^{\circ} \mathrm{C}$ during our acute 

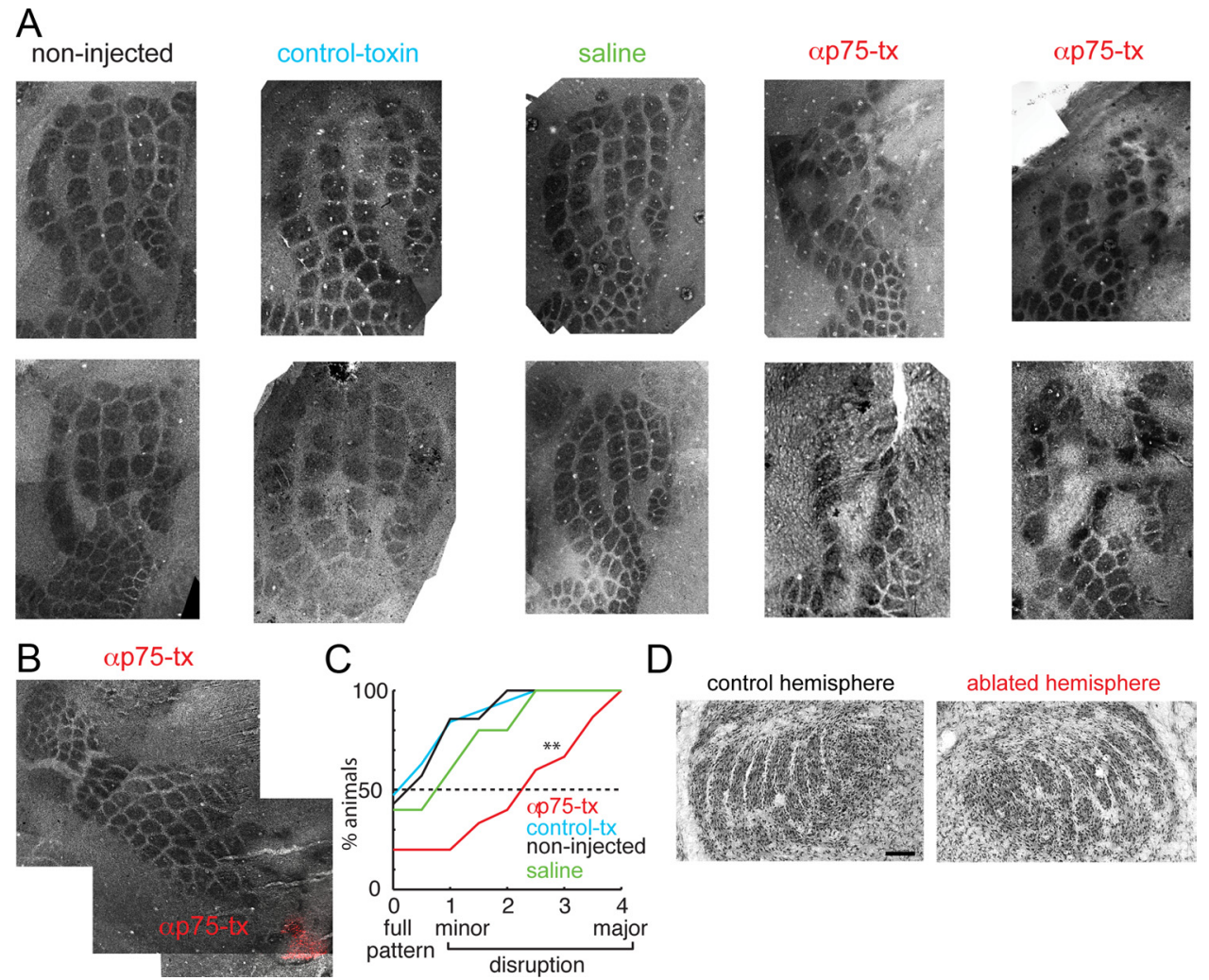

Figure 5. Subplate neurons are required for $S 1$ barrel formation. $\boldsymbol{A}$, Merged images of cytochrome oxidase-stained sections from (from left to right): 2 control non-injected animals, 2 animals injected with control toxin ( $\mu$-toxin), 2 animals injected with saline, and 4 animals injected with anti-rat p75-immunotoxin. In anti-rat p 75-immunotoxin-injected animals clear gaps were present in the barrel field. B, Cytochrome oxidase-stained sections from an anti-rat p75-immunotoxin-injected animal in which fluorescent beads (red) were overlaid on the bright-field image. Note that barrels in vicinity to fluorescent beads were disrupted. C, Quantification of the ablation effect shown as a cumulative distribution of disruption scores for animals in the four experimental categories. A score of 0 indicates the presence of a full barrel field while scores from 1 to 4 indicate progressively more severe disruptions. Plotted is the fraction of animals for each manipulation category. Injections of p75-immunotoxin (red) caused a disruption of the barrel pattern compared with control toxin (black) or non-injected control (blue) (both $p<0.05$ ). Injections of saline (green) or control toxin (black) did not cause a significant disruption $(p>0.1)$. D. Cytochrome oxidase-stained thalamic sections from an ablated and non-injected control hemisphere showing the barreloids. No obvious differences are seen. Scale bar, $100 \mu \mathrm{m}$.

and chronic experiments may have caused a generally lower level of spindle burst and sharp potential activity (Karlsson and Blumberg, 2004). Anesthesia can also affect spindle bursts (Colonnese et al., 2010). However, we find that the effects of SPN ablation are comparable for both the acute and chronic experiments, thus potential confounding effects in the acute experiments of recovery from anesthesia seem to be minimal.

The development of thalamocortical patterning in both rodent somatosensory barrel cortex and ocular dominance columns (ODCs) in cat visual cortex is thought to be dependent on neuronal activity (Chapman et al., 1986; Schlaggar et al., 1993; O'Leary et al., 1994; Erzurumlu and Kind, 2001; Sengpiel and Kind, 2002; Feldman and Brecht, 2005; Inan and Crair, 2007) and mechanisms of synaptic plasticity mediated by NMDA receptors (Fox et al., 1996; Iwasato et al., 2000). SPN removal in cat prevents the formation of ODCs (Ghosh and Shatz, 1992; Kanold et al., 2003), and our data here show that SPN removal in rodent prevents the formation and refinement of the barrel pattern. SPN neurites have also been shown to be dynamically integrated into the developing barrel cortex circuitry (Piñon et al., 2009). Thus SPNs seem to play functionally similar roles in cat and rodents and across cortical areas. The observed variability in the effect of subplate removal on the barrel pattern might be due to variability in the positioning of our injections and therefore the extent of the ablation. Moreover, we observed that in subplate-ablated regions cells located in areas with a larger concentration of fluorescent beads (that were coinjected with the immunotoxin) tended to show smaller maximal EPSCs suggesting a graded effect of the ablation. In addition, the variability in the barrel pattern could be due to variability of the maturity of the barrel pattern at time of ablation. We performed our ablations at $\mathrm{P} 0$, which may coincide with the end of the time window for the role of the subplate in barrel formation. This notion is supported by the fact that subplate damage via neonatal hypoxia-ischemia at $\mathrm{P} 1 / \mathrm{P} 2$ seems to not interfere with barrel formation (McQuillen et al., 2003), but rather with barrel plasticity (Failor et al., 2006; Quairiaux et al., 2010). Finally, the disruption in the barrel pattern that we observed using cytochrome oxidase staining could also at least partially reflect the decreased sensory driven activity in the cortex since cytochrome oxidase levels can be regulated by neuronal activity.

Our data suggest that SPNs play an important developmental role in the functional and structural development of the S1 cortex. However in all neuronal ablation studies it is possible that some of the observed effects could be partially mediated by pathways other than the loss of the targeted neurons themselves or the loss of neural activity. SPN immunolesions may be accompanied by microglial or glial activation (Book et al., 1995) with possible consequent non-neural activity-dependent effects on brain development and plasticity (Roumier et al., 2008; Tremblay et al., 2010). However, since layer 4 cells were physiologically normal consequent to SPN ablation, our results suggest that such possi- 
ble effects did not spread to this layer. On the other hand, cortical activity itself can affect microglial activation thus effects of SPN removal on neuronal activity can change the status of glia (Tremblay et al., 2010; Fontainhas et al., 2011). In addition, SPNs selectively express connective tissue growth factor and are coupled via gap junctions (Heuer et al., 2003; Kanold and Luhmann, 2010; Wang et al., 2011) suggesting that SPNs might signal to nonneuronal cells, and/or signal to other neurons not only via chemical synapses. Thus while the effects of SPN removal are consistent with a neural signaling role of SPNs via their excitatory synapses with layer 4 , it is possible that some effects of SPN removal are mediated by other pathways, possibly involving nonneuronal cells. Recent identification of a host of subplate-specific genes (Hoerder-Suabedissen et al., 2009; Osheroff and Hatten, 2009; Oeschger et al., 2011) might enable the development of methods to dissect the role of SPN-mediated neuronal activity and other potential signaling routes of SPNs.

In newborn preterm and term babies, observation of spontaneous movements is used to assess neurological development, whereby abnormalities have been linked to damage of periventricular regions that include the subplate (Volpe, 1996; Bos et al., 1998; Hadders-Algra, 2007). Functional data from human subplate are scarce, and direct information is available mostly from in vitro studies (Moore et al., 2011). Therefore the altered EEG pattern of local subplate damage in rodents, leading to a loss of the characteristic spindle burst activity, may help to identify EEG patterns indicative of local cortical or white matter damage in human babies. Thus the spontaneous and sensory evoked activity patterns in the EEG in human babies might be used for early neurological assessment (Vanhatalo et al., 2009; Vanhatalo and Kaila, 2010).

In summary, we show here that SPNs play an important role in the generation of spindle bursts. Neuronal activity is required for the normal development of cortical circuits, and the present work shows that the spindle burst network activity promoted by SPNs is in a key position to affect cortical development.

\section{References}

Adelsberger H, Garaschuk O, Konnerth A (2005) Cortical calcium waves in resting newborn mice. Nat Neurosci 8:988-990.

Agmon A, Connors BW (1991) Thalamocortical responses of mouse somatosensory (barrel) cortex in vitro. Neuroscience 41:365-379.

Ben-Ari Y (2001) Developing networks play a similar melody. Trends Neurosci 24:353-360.

Bernard C, Milh M, Morozov YM, Ben-Ari Y, Freund TF, Gozlan H (2005) Altering cannabinoid signaling during development disrupts neuronal activity. Proc Natl Acad Sci U S A 102:9388-9393.

Book AA, Wiley RG, Schweitzer JB (1995) 192 IgG-saporin. 2. Neuropathology in the rat brain. Acta Neuropathol 89:519-526.

Bos AF, Martijn A, Okken A, Prechtl HF (1998) Quality of general movements in preterm infants with transient periventricular echodensities. Acta Paediatr 87:328-335.

Calderon DP, Leverkova N, Peinado A (2005) Gq/11-induced and spontaneous waves of coordinated network activation in developing frontal cortex. J Neurosci 25:1737-1749.

Chapman B, Jacobson MD, Reiter HO, Stryker MP (1986) Ocular dominance shift in kitten visual cortex caused by imbalance in retinal electrical activity. Nature 324:154-156.

Colonnese MT, Khazipov R (2010) "Slow activity transients" in infant rat visual cortex: a spreading synchronous oscillation patterned by retinal waves. J Neurosci 30:4325-4337.

Colonnese MT, Kaminska A, Minlebaev M, Milh M, Bloem B, Lescure S, Moriette G, Chiron C, Ben-Ari Y, Khazipov R (2010) A conserved switch in sensory processing prepares developing neocortex for vision. Neuron 67:480-498.

Dupont E, Hanganu IL, Kilb W, Hirsch S, Luhmann HJ (2006) Rapid devel- opmental switch in the mechanisms driving early cortical columnar networks. Nature 439:79-83.

Erzurumlu RS, Kind PC (2001) Neural activity: sculptor of 'barrels' in the neocortex. Trends Neurosci 24:589-595.

Evrard A, Ropert N (2009) Early development of the thalamic inhibitory feedback loop in the primary somatosensory system of the newborn mice. J Neurosci 29:9930-9940.

Failor S, Evans M, Cang J, Stryker M, McQuillen P (2006) Impaired cortical plasticity after early hypoxia-ischemia. Soc Neurosci Abstr 32:717.14.

Feldman DE, Brecht M (2005) Map plasticity in somatosensory cortex. Science 310:810-815.

Fontainhas AM, Wang M, Liang KJ, Chen S, Mettu P, Damani M, Fariss RN, Li W, Wong WT (2011) Microglial morphology and dynamic behavior is regulated by ionotropic glutamatergic and GABAergic neurotransmission. PLoS One 6:e15973.

Fox K, Schlaggar BL, Glazewski S, O’Leary DD (1996) Glutamate receptor blockade at cortical synapses disrupts development of thalamocortical and columnar organization in somatosensory cortex. Proc Natl Acad Sci U S A 93:5584-5589.

Friauf E, Shatz CJ (1991) Changing patterns of synaptic input to subplate and cortical plate during development of visual cortex. J Neurophysiol 66:2059-2071.

Garaschuk O, Linn J, Eilers J, Konnerth A (2000) Large-scale oscillatory calcium waves in the immature cortex. Nat Neurosci 3:452-459.

Ghosh A, Shatz CJ (1992) Involvement of subplate neurons in the formation of ocular dominance columns. Science 255:1441-1443.

Gonzalez-Islas C, Wenner P (2006) Spontaneous network activity in the embryonic spinal cord regulates AMPAergic and GABAergic synaptic strength. Neuron 49:563-575.

Hadders-Algra M (2007) Putative neural substrate of normal and abnormal general movements. Neurosci Biobehav Rev 31:1181-1190.

Hanganu IL, Kilb W, Luhmann HJ (2002) Functional synaptic projections onto subplate neurons in neonatal rat somatosensory cortex. J Neurosci 22:7165-7176.

Hanganu IL, Ben-Ari Y, Khazipov R (2006) Retinal waves trigger spindle bursts in the neonatal rat visual cortex. J Neurosci 26:6728-6736.

Heuer H, Christ S, Friedrichsen S, Brauer D, Winckler M, Bauer K, Raivich G (2003) Connective tissue growth factor: a novel marker of layer VII neurons in the rat cerebral cortex. Neuroscience 119:43-52.

Hirsch S, Luhmann HJ (2008) Pathway-specificity in N-methyl-D-aspartate receptor-mediated synaptic inputs onto subplate neurons. Neuroscience 153:1092-1102.

Hoerder-Suabedissen A, Wang WZ, Lee S, Davies KE, Goffinet AM, Rakić S, Parnavelas J, Reim K, Nicolić M, Paulsen O, Molnár Z (2009) Novel markers reveal subpopulations of subplate neurons in the murine cerebral cortex. Cereb Cortex 19:1738-1750.

Inan M, Crair MC (2007) Development of cortical maps: perspectives from the barrel cortex. Neuroscientist 13:49-61.

Iwasato T, Datwani A, Wolf AM, Nishiyama H, Taguchi Y, Tonegawa S, Knöpfel T, Erzurumlu RS, Itohara S (2000) Cortex-restricted disruption of NMDAR1 impairs neuronal patterns in the barrel cortex. Nature 406:726-731.

Kanold PO, Luhmann HJ (2010) The subplate and early cortical circuits. Annu Rev Neurosci 33:23-48.

Kanold PO, Shatz CJ (2006) Subplate neurons regulate maturation of cortical inhibition and outcome of ocular dominance plasticity. Neuron 51:627-638.

Kanold PO, Kara P, Reid RC, Shatz CJ (2003) Role of subplate neurons in functional maturation of visual cortical columns. Science 301:521-525.

Karlsson KA, Blumberg MS (2004) Temperature-induced reciprocal activation of hippocampal field activity. J Neurophysiol 91:583-588.

Katz LC, Shatz CJ (1996) Synaptic activity and the construction of cortical circuits. Science 274:1133-1138.

Khazipov R, Sirota A, Leinekugel X, Holmes GL, Ben-Ari Y, Buzsáki G (2004) Early motor activity drives spindle bursts in the developing somatosensory cortex. Nature 432:758-761.

Koh S, Loy R (1989) Localization and development of nerve growth factorsensitive rat basal forebrain neurons and their afferent projections to hippocampus and neocortex. J Neurosci 9:2999-3018.

Kostovic I, Rakic P (1980) Cytology and time of origin of interstitial neurons in the white matter in infant and adult human and monkey telencephalon. J Neurocytol 9:219-242. 
Marcano-Reik AJ, Blumberg MS (2008) The corpus callosum modulates spindle-burst activity within homotopic regions of somatosensory cortex in newborn rats. Eur J Neurosci 28:1457-1466.

Marcano-Reik AJ, Prasad T, Weiner JA, Blumberg MS (2010) An abrupt developmental shift in callosal modulation of sleep-related spindle bursts coincides with the emergence of excitatory-inhibitory balance and a reduction of somatosensory cortical plasticity. Behav Neurosci 124:600-611.

McQuillen PS, Sheldon RA, Shatz CJ, Ferriero DM (2003) Selective vulnerability of subplate neurons after early neonatal hypoxia-ischemia. J Neurosci 23:3308-3315.

Milh M, Kaminska A, Huon C, Lapillonne A, Ben-Ari Y, Khazipov R (2007) Rapid cortical oscillations and early motor activity in premature human neonate. Cereb Cortex 17:1582-1594.

Minlebaev M, Ben-Ari Y, Khazipov R (2007) Network mechanisms of spindle-burst oscillations in the neonatal rat barrel cortex in vivo. J Neurophysiol 97:692-700.

Minlebaev M, Ben-Ari Y, Khazipov R (2009) NMDA receptors pattern early activity in the developing barrel cortex in vivo. Cereb Cortex 19:688-696.

Mohns EJ, Blumberg MS (2010) Neocortical activation of the hippocampus during sleep in infant rats. J Neurosci 30:3438-3449.

Moore AR, Zhou WL, Jakovcevski I, Zecevic N, Antic SD (2011) Spontaneous electrical activity in the human fetal cortex in vitro. J Neurosci 31:2391-2398.

Oeschger FM, Wang WZ, Lee S, García-Moreno F, Goffinet AM, Arbonés ML, Rakic S, Molnár Z (2011) Gene expression analysis of the embryonic subplate. Cereb Cortex. Advance online publication. Retrieved October 24, 2011. doi:10.1093/cercor/bhr197.

O’Leary DD, Schlaggar BL, Tuttle R (1994) Specification of neocortical areas and thalamocortical connections. Annu Rev Neurosci 17:419-439.

Osheroff H, Hatten ME (2009) Gene expression profiling of preplate neurons destined for the subplate: genes involved in transcription, axon extension, neurotransmitter regulation, steroid hormone signaling, and neuronal survival. Cereb Cortex 19 [Suppl 1]:i126-i134.

Piñon MC, Jethwa A, Jacobs E, Campagnoni A, Molnár Z (2009) Dynamic integration of subplate neurons into the cortical barrel field circuitry during postnatal development in the Golli-tau-eGFP (GTE) mouse. J Physiol 587:1903-1915.

Quairiaux C, Sizonenko SV, Mégevand P, Michel CM, Kiss JZ (2010) Functional deficit and recovery of developing sensorimotor networks following neonatal hypoxic-ischemic injury in the rat. Cereb Cortex 20:2080-2091.

Rheims S, Minlebaev M, Ivanov A, Represa A, Khazipov R, Holmes GL, Ben-Ari Y, Zilberter Y (2008) Excitatory GABA in rodent developing neocortex in vitro. J Neurophysiol 100:609-619.

Roumier A, Pascual O, Béchade C, Wakselman S, Poncer JC, Réal E, Triller A, Bessis A (2008) Prenatal activation of microglia induces delayed impairment of glutamatergic synaptic function. PLoS One 3:e2595.

Ruusuvuori E, Li H, Huttu K, Palva JM, Smirnov S, Rivera C, Kaila K, Voipio J (2004) Carbonic anhydrase isoform VII acts as a molecular switch in the development of synchronous gamma-frequency firing of hippocampal CA1 pyramidal cells. J Neurosci 24:2699-2707.

Schlaggar BL, Fox K, O'Leary DD (1993) Postsynaptic control of plasticity in developing somatosensory cortex. Nature 364:623-626.

Schuchmann S, Schmitz D, Rivera C, Vanhatalo S, Salmen B, Mackie K, Sipilä ST, Voipio J, Kaila K (2006) Experimental febrile seizures are precipitated by a hyperthermia-induced respiratory alkalosis. Nat Med 12:817-823.
Seelke AM, Blumberg MS (2010) Developmental appearance and disappearance of cortical events and oscillations in infant rats. Brain Res 1324:34-42.

Sengpiel F, Kind PC (2002) The role of activity in development of the visual system. Curr Biol 12:R818-R826.

Sipilä ST, Schuchmann S, Voipio J, Yamada J, Kaila K (2006) The cationchloride cotransporter NKCC1 promotes sharp waves in the neonatal rat hippocampus. J Physiol 573:765-773.

Sun JJ, Luhmann HJ (2007) Spatio-temporal dynamics of oscillatory network activity in the neonatal mouse cerebral cortex. Eur J Neurosci 26:1995-2004.

Tallgren P, Vanhatalo S, Kaila K, Voipio J (2005) Evaluation of commercially available electrodes and gels for recording of slow EEG potentials. Clin Neurophysiol 116:799-806.

Tolner EA, van Vliet EA, Holtmaat AJ, Aronica E, Witter MP, da Silva FH, Gorter JA (2003) GAP-43 mRNA and protein expression in the hippocampal and parahippocampal region during the course of epileptogenesis in rats. Eur J Neurosci 17:2369-2380.

Tremblay MÈ, Lowery RL, Majewska AK (2010) Microglial interactions with synapses are modulated by visual experience. PLoS Biol 8:e1000527.

Vanhatalo S, Kaila K (2010) Emergence of spontaneous and evoked electroencephalographic activity in the human brain. In: The newborn brain: neuroscience and clinical applications, Ed 2 (Lagercrantz H, Hanson M, Evrard P, Rod C, eds), pp 229-244. Cambridge, UK: Cambridge UP.

Vanhatalo S, Tallgren P, Andersson S, Sainio K, Voipio J, Kaila K (2002) DC-EEG discloses prominent, very slow activity patterns during sleep in preterm infants. Clin Neurophysiol 113:1822-1825.

Vanhatalo S, Palva JM, Andersson S, Rivera C, Voipio J, Kaila K (2005) Slow endogenous activity transients and developmental expression of $\mathrm{K}^{+}-\mathrm{Cl}^{-}$cotransporter 2 in the immature human cortex. Eur J Neurosci 22:2799-2804.

Vanhatalo S, Jousmäki V, Andersson S, Metsäranta M (2009) An easy and practical method for routine, bedside testing of somatosensory systems in extremely low birth weight infants. Pediatr Res 66:710-713.

Volpe JJ (1996) Subplate neurons-missing link in brain injury of the premature infant? Pediatrics 97:112-113.

Wang WZ, Oeschger FM, Montiel JF, García-Moreno F, Hoerder-Suabedissen A, Krubitzer L, Ek CJ, Saunders NR, Reim K, Villalón A, Molnár Z (2011) Comparative aspects of subplate zone studied with gene expression in sauropsids and mammals. Cereb Cortex 21:2187-2203.

Xu HP, Furman M, Mineur YS, Chen H, King SL, Zenisek D, Zhou ZJ, Butts DA, Tian N, Picciotto MR, Crair MC (2011) An instructive role for patterned spontaneous retinal activity in mouse visual map development. Neuron 70:1115-1127.

Yang JW, Hanganu-Opatz IL, Sun JJ, Luhmann HJ (2009) Three patterns of oscillatory activity differentially synchronize developing neocortical networks in vivo. J Neurosci 29:9011-9025.

Yuste R, Denk W (1995) Dendritic spines as basic functional units of neuronal integration. Nature 375:682-684.

Zhang LI, Poo MM (2001) Electrical activity and development of neural circuits. Nat Neurosci 4 [Suppl]:1207-1214.

Zhao C, Kao JP, Kanold PO (2009) Functional excitatory microcircuits in neonatal cortex connect thalamus and layer 4. J Neurosci 29:1547915488. 